\title{
Generalized Pointwise Hölder Spaces Defined via Admissible Sequences
}

\author{
Damien Kreit and Samuel Nicolay (1) \\ Université de Liège, Institut de Mathématique, 12 Allée de la Découverte, Bâtiment B37, Sart-Tilman, 4000 Liège, Belgium \\ Correspondence should be addressed to Samuel Nicolay; s.nicolay@uliege.be
}

Received 29 January 2018; Revised 29 March 2018; Accepted 8 April 2018; Published 29 May 2018

Academic Editor: Adrian Petrusel

Copyright (c) 2018 Damien Kreit and Samuel Nicolay. This is an open access article distributed under the Creative Commons Attribution License, which permits unrestricted use, distribution, and reproduction in any medium, provided the original work is properly cited.

We introduce in this paper a generalization of the pointwise Hölder spaces. We give alternative definitions of these spaces, look at their relationship with the wavelets, and introduce a notion of generalized Hölder exponent.

\section{Introduction}

A real-valued function $f$ defined on $\mathbf{R}^{d}$ belongs to the uniform Hölder space $\Lambda^{\alpha}\left(\mathbf{R}^{d}\right)(\alpha>0)$ if there exist a constant $C$ and a polynomial $P$ of degree less than $\alpha$ such that

$$
|f(x+h)-P(x)| \leq C|h|^{\alpha},
$$

for all $x \in \mathbf{R}^{d}$ and $h$ small enough. The supremum of all these values $\alpha$ is called the global Hölder exponent of $f$. One can naturally define the pointwise version of these spaces: a continuous function $f$ belongs to the pointwise Hölder space $\Lambda^{\alpha}\left(x_{0}\right)$ if and only if there exist $C, J>0$ and a polynomial $P$ of degree less than $\alpha$ such that

$$
\sup _{|h| \leq 2^{-j}}\left|f\left(x_{0}+h\right)-P(h)\right| \leq C 2^{-j \alpha}
$$

for all $j \geq J$. Of course, the supremum of all these values $\alpha$ is called the Hölder exponent of $f$ at $x_{0}$. If $f$ is differentiable at $x_{0}$, the Hölder exponent of $f$ at $x_{0}$ is at least 1 . The fact that the constant $C$ appearing in (1) is uniform for $\Lambda^{\alpha}\left(\mathbf{R}^{d}\right)$ implies that the uniform Hölder exponent is not necessarily the infimum of the pointwise Hölder exponents. A classical example is the function $\operatorname{sinc}(1 / x)=x \sin (1 / x)$, which is $C^{1}$ at the origin, $C^{\infty}$ everywhere else, while its global Hölder exponent is $1 / 2$.

In $[1,2]$, the properties of generalized uniform Hölder spaces have been investigated. The idea underlying the definition is to replace the exponent $\alpha$ of the usual spaces
$\Lambda^{\alpha}\left(\mathbf{R}^{d}\right)[3,4]$ with a sequence $\sigma$ satisfying some conditions. The so-obtained spaces $\Lambda^{\sigma}\left(\mathbf{R}^{d}\right)$ generalize the spaces $\Lambda^{\alpha}\left(\mathbf{R}^{d}\right)$; the spaces $\Lambda^{\sigma}\left(\mathbf{R}^{d}\right)$ are actually the spaces $B_{\infty, \infty}^{1 / \sigma}\left(\mathbf{R}^{d}\right)$, but they present specific properties (induced by $L^{\infty}$-norms) when compared to the more general spaces $B_{p, q}^{1 / \sigma}\left(\mathbf{R}^{d}\right)$ studied in [5$10]$, for example. Indeed, it is shown in $[1,2]$ that most of the usual properties holding for the spaces $\Lambda^{\alpha}\left(\mathbf{R}^{d}\right)$ can be transposed to the spaces $\Lambda^{\sigma}\left(\mathbf{R}^{d}\right)$.

Here, we introduce the pointwise version of these spaces: the spaces $\Lambda^{\sigma, M}\left(x_{0}\right)$, with $x_{0} \in \mathbf{R}^{d}$. As in $[1,2]$, the idea is again to replace the sequence $\left(2^{-j \alpha}\right)_{j}$ appearing in (2) with a positive sequence $\left(\sigma_{j}\right)_{j}$ such that $\sigma_{j+1} / \sigma_{j}$ and $\sigma_{j} / \sigma_{j+1}$ are bounded (for any $j$ ); the number $M$ stands for the maximal degree of the polynomial (this degree cannot be induced by a sequence $\sigma$ ). By doing so, one tries to get a better characterization of the regularity of the studied function $f$; a usual choice is to replace $2^{-j \alpha}$ with $j 2^{-j \alpha}$ (see, e.g., [11-13]). For example, it is well known that, for the Brownian motion $B$, there exist $C, J>0$ such that

$$
\sup _{|h| \leq 2^{-j}}|B(x+h)-B(x)| \leq C \sqrt{j} 2^{-j / 2},
$$

for any $j \geq J$ almost surely (see, e.g., $[14,15])$. More generally, the behavior of the oscillations of $f(x+h)-f(x)$ as $h$ tends to 0 can reveal specific local behaviors such as approximate similarities [11]. Generalizations of the pointwise Hölder spaces have already been proposed, but, to our knowledge, 
the definition we give here is the most general version and leads to the sharpest results (in particular, the regularity spaces introduced in [13] are a special case of the spaces $\Lambda^{\sigma, M}\left(x_{0}\right)$; see Definition 2 and Remark 4$)$. These spaces could also be useful in the study of singularities of PDEs where the function is smooth near $x_{0}$ except on small sets of points (such a situation could be met in the case of the Navier-Stokes equations in three dimensions [16]).

As a first application, we introduce here the sufficient tools to develop a multifractal formalism based on the wavelet characterization (Theorem 16), in the same spirit as in [12]. The theory presented here also contributes to enlightening the reasons why the customary proofs for the classical spaces $\Lambda^{\alpha}\left(x_{0}\right)$ work.

This paper is organized as follows. We first give the definitions leading to generalized pointwise Hölder spaces $\Lambda^{\sigma, M}\left(x_{0}\right)$ and prove that, under some general conditions, the polynomials appearing in the definition are independent of the scale, as is the case with the usual Hölder spaces. Next, we give some alternative definitions of the spaces $\Lambda^{\sigma, M}\left(x_{0}\right)$, mimicking the different possible definitions of $\Lambda^{\alpha}\left(x_{0}\right)$. One of the nicest properties of the Hölder spaces is their relationship with the wavelet theory given in [12]; we show here that this result still holds in the general case. Finally, we give some conditions under which one gets embedded generalized pointwise Hölder spaces and define a generalized pointwise Hölder exponent.

Throughout this paper, $B$ denotes the open unit ball of $\mathbf{R}^{d}$ centered at the origin; moreover, we set $B_{j}=2^{-j} B$. The floor function is denoted by $[\cdot]$ and $\mathbf{P}[\alpha]$ designates the set of polynomials of degree at most $[\alpha]$. We use the letter $C$ for a generic positive constant whose value may be different at each occurrence.

\section{Pointwise Generalized Hölder Spaces}

To present the generalized pointwise Hölder spaces, we first need to recall some notions concerning the admissible sequences. After having introduced the definitions, we point out a major difference between the usual spaces and the generalized ones: the polynomial arising in the definition depends on the scale. It is then natural to look under which conditions this constraint can be dropped.

2.1. Definition. The generalization of the Hölder spaces we propose here is based on the notion of admissible sequence [10].

Definition 1. A sequence $\sigma=\left(\sigma_{j}\right)_{j \in \mathrm{N}}$ of real positive numbers is called admissible if there exists a positive constant $C$ such that

$$
C^{-1} \sigma_{j} \leq \sigma_{j+1} \leq C \sigma_{j}
$$

for any $j \in \mathbf{N}$.
If $\sigma$ is such a sequence, we set

$$
\begin{aligned}
& \underline{\Theta}_{j}=\inf _{k \in \mathbf{N}} \frac{\sigma_{j+k}}{\sigma_{k}}, \\
& \bar{\Theta}_{j}=\sup _{k \in \mathbf{N}} \frac{\sigma_{j+k}}{\sigma_{k}},
\end{aligned}
$$

and we define the lower and upper Boyd indices as follows:

$$
\begin{aligned}
& \underline{s}(\sigma)=\lim _{j} \frac{\log _{2} \underline{\Theta}_{j}}{j}, \\
& \bar{s}(\sigma)=\lim _{j} \frac{\log _{2} \bar{\Theta}_{j}}{j} .
\end{aligned}
$$

Since $\left(\log \underline{\Theta}_{j}\right)_{j \in \mathbf{N}}$ is a subadditive sequence, such limits always exist [17]. The following relations about such sequences are well known (see, e.g., [1]). If $\sigma$ is an admissible sequence, let $\varepsilon>0$; there exists a positive constant $C$ such that

$$
C^{-1} 2^{j(\underline{s}(\sigma)-\varepsilon)} \leq \underline{\Theta}_{j} \leq \frac{\sigma_{j+k}}{\sigma_{k}} \leq \bar{\Theta}_{j} \leq C 2^{j(\bar{s}(\sigma)+\varepsilon)},
$$

for any $j, k \in \mathbf{N}$. Let $\sigma$ be an admissible sequence:

(i) if $\underline{s}(\sigma)>0$, then there exists a positive constant $C$ such that, for any $J \in \mathbf{N}$,

$$
\sum_{j=J}^{\infty} \sigma_{j}^{-1} \leq C \sigma_{J}^{-1}
$$

(ii) if $n \in \mathbf{N}$ satisfies $\bar{s}(\sigma)<n$, then there exists a positive constant $C$ such that, for any $J \in \mathbf{N}$,

$$
\sum_{j=1}^{J} 2^{j n} \sigma_{j}^{-1} \leq C 2^{J n} \sigma_{J}^{-1}
$$

In this paper, $\sigma$ will always stand for an admissible sequence and $M$ for a natural number, possibly zero.

Starting from the definitions of the pointwise Hölder spaces $\Lambda^{\alpha}\left(x_{0}\right)$ (with $\alpha>0$ ) and the generalized uniform Hölder spaces $\Lambda^{\sigma}\left(\mathbf{R}^{d}\right)$ introduced in [1], we are naturally led to the following definition.

Definition 2. Let $x_{0} \in \mathbf{R}^{d}$; a continuous function $f$ belongs to $\Lambda^{\sigma, M}\left(x_{0}\right)$ if there exist $C, J>0$ such that

$$
\inf _{P \in \mathbf{P}[M]}\|f-P\|_{L^{\infty}\left(x_{0}+B_{j}\right)} \leq C \sigma_{j},
$$

for any $j \geq J$.

We trivially have the following alternative definition for $\Lambda^{\sigma, M}\left(x_{0}\right)$.

Definition 3. A continuous function $f$ belongs to $\Lambda^{\sigma, M}\left(x_{0}\right)$ if there exist $C, J>0$ such that, for any $j \geq J$, there exists a polynomial $P_{j} \in \mathbf{P}[M]$ for which

$$
\sup _{h \in B_{j}}\left|f\left(x_{0}+h\right)-P_{j}\left(x_{0}+h\right)\right| \leq C \sigma_{j} .
$$


Remark 4. In [13], a generalization of the pointwise Hölder spaces is introduced by replacing the admissible sequence appearing in Definition 2 by a modulus of smoothness (more precisely, the definition is based on (34)). As shown in [1], given a modulus of smoothness $\omega, \sigma_{j}=\omega\left(2^{-j}\right)$ defines an admissible sequence. The converse is not necessarily true: only the decreasing admissible sequences converging to 0 give rise to a modulus of continuity [1].

Sometimes, we will also need to impose a slightly stronger condition than continuity to the function.

Definition 5. A function $f$ is uniformly Hölder if there exists $\varepsilon>0$ such that $f \in \Lambda^{\varepsilon}\left(\mathbf{R}^{d}\right)$.

2.2. Independence of the Polynomial from the Scale. It is important to remark that the polynomial occurring in inequality (11) is a function of the scale $j$. However, for the classical Hölder spaces, such polynomial is independent of $j$. Here, we look under which conditions the independence still holds in the generalized case, that is, under which conditions $P_{j}=P \in \mathbf{P}[M]$ for any $j \geq J$. In this section, $f$ will designate a continuous function of $L_{\mathrm{loc}}^{\infty}\left(\mathbf{R}^{d}\right)$ (although the continuity hypothesis is very often dropped when dealing with $L^{\infty}$ norms, we keep it here to ensure the equivalence with Definition 3).

We will need the following Markov inequality (see, e.g., [18]): Let $p \in(0, \infty], k \in\{1, \ldots, d\}$, and $S \subset \mathbf{R}^{d}$ be a bounded convex set with nonempty interior; one has

$$
\left\|D_{k} P\right\|_{L^{p}(S)} \leq C n^{2}\|P\|_{L^{p}(S)},
$$

for any $P \in \mathbf{P}[n]$, where $D_{k} P$ denotes the partial derivative of $P$ following the $k$ th variable and where $C$ depends only on $S$ and $p$. If $x_{0} \in \mathbf{R}^{d}$, we thus have

$$
\left\|D_{k} P\right\|_{L^{\infty}\left(x_{0}+r B\right)} \leq \frac{C n^{2}}{r}\|P\|_{L^{\infty}\left(x_{0}+r B\right)},
$$

for any $r>0$ and any $P \in \mathbf{P}[n]$, where $C$ is a constant (and does not depend on $P, n$, or $r$ ).

Lemma 6. If $f \in \Lambda^{\sigma, M}\left(x_{0}\right)$ with $M<\underline{s}\left(\sigma^{-1}\right)$, the sequence of polynomials $\left(P_{j}\right)_{j}$ occurring in (11) satisfies

$$
\left\|D^{\beta} P_{k}-D^{\beta} P_{j}\right\|_{L^{\infty}\left(x_{0}+B_{k}\right)} \leq C 2^{j|\beta|} \sigma_{j},
$$

for any multi-index $\beta$ such that $|\beta| \leq M$ and $k \geq j \geq J$.

In particular, $\left(D^{\beta} P_{j}\left(x_{0}\right)\right)_{j}$ is a Cauchy sequence for any multi-index $\beta$ such that $|\beta| \leq M$.

Proof. Using the Markov inequality, we get

$$
\begin{aligned}
& \left\|D^{\beta} P_{j}-D^{\beta} P_{j+1}\right\|_{L^{\infty}\left(x_{0}+B_{j+1}\right)} \\
& \leq C 2^{|\beta| j}\left\|P_{j}-P_{j+1}\right\|_{L^{\infty}\left(x_{0}+B_{j+1}\right)} \\
& \leq C 2^{|\beta| j}\left(\left\|P_{j}-f\right\|_{L^{\infty}\left(x_{0}+B_{j+1}\right)}+\left\|f-P_{j+1}\right\|_{L^{\infty}\left(x_{0}+B_{j+1}\right)}\right) \\
& \leq C 2^{|\beta| j}\left(\sigma_{j}+\sigma_{j+1}\right) \leq C^{\prime} 2^{|\beta| j} \sigma_{j},
\end{aligned}
$$

for any $\beta$ such that $|\beta| \leq M$. Therefore, if $k$ satisfies $k \geq j \geq J$, one gets

$$
\begin{aligned}
& \left\|D^{\beta}\left(P_{j}-P_{k}\right)\right\|_{L^{\infty}\left(x_{0}+B_{k}\right)} \\
& \leq \sum_{l=j}^{k-1}\left\|D^{\beta}\left(P_{l}-P_{l+1}\right)\right\|_{L^{\infty}\left(x_{0}+B_{k}\right)} \\
& \quad \leq \sum_{l=j}^{k-1}\left\|D^{\beta}\left(P_{l}-P_{l+1}\right)\right\|_{L^{\infty}\left(x_{0}+B_{l+1}\right)} \leq C \sum_{l=j}^{k-1} 2^{|\beta| l} \sigma_{l} \\
& \leq C 2^{|\beta| j} \sigma_{j},
\end{aligned}
$$

which is the desired result.

Lemma 7. If $f \in \Lambda^{\sigma, M}\left(x_{0}\right)$ with $M<\underline{s}\left(\sigma^{-1}\right)$ and $\left(P_{j}\right)_{j}$ is a sequence of polynomials satisfying inequality (11), then, for any multi-index $\beta$ such that $|\beta| \leq M$, the limit

$$
f_{\beta}\left(x_{0}\right)=\lim _{j \rightarrow \infty} D^{\beta} P_{j}\left(x_{0}\right)
$$

is independent of the chosen sequence $\left(P_{j}\right)_{j}$.

Proof. If $\left(Q_{j}\right)_{j}$ is another sequence of polynomials satisfying inequality (11), one gets

$$
\begin{aligned}
& \left|D^{\beta} Q_{j}\left(x_{0}\right)-f_{\beta}\left(x_{0}\right)\right| \\
& \leq\left|D^{\beta} Q_{j}\left(x_{0}\right)-D^{\beta} P_{j}\left(x_{0}\right)\right| \\
& \quad+\left|D^{\beta} P_{j}\left(x_{0}\right)-f_{\beta}\left(x_{0}\right)\right| .
\end{aligned}
$$

One has, using the Markov inequality,

$$
\begin{aligned}
& \left\|D^{\beta}\left(P_{j}-Q_{j}\right)\right\|_{L^{\infty}\left(x_{0}+B_{j}\right)} \leq C 2^{|\beta| j}\left\|P_{j}-Q_{j}\right\|_{L^{\infty}\left(x_{0}+B_{j}\right)} \\
& \quad \leq C 2^{|\beta| j}\left(\left\|P_{j}-f\right\|_{L^{\infty}\left(x_{0}+B_{j}\right)}+\left\|f-Q_{j}\right\|_{L^{\infty}\left(x_{0}+B_{j}\right)}\right) \\
& \quad \leq C 2^{|\beta| j} \sigma_{j} \longrightarrow 0,
\end{aligned}
$$

as $j \rightarrow \infty$, which ends the proof.

For such functions, we can introduce the notion of Peano derivative (see, e.g., $[13,19]$ for more information).

Definition 8. Under the hypothesis of Lemma 7, the $\beta$ th Peano derivative of $f$ at $x_{0}$ is $f_{\beta}\left(x_{0}\right)=\lim _{j} D^{\beta} P_{j}\left(x_{0}\right)$.

We can now obtain the result concerning the independence of the polynomials.

Theorem 9. If $M<\underline{s}\left(\sigma^{-1}\right)$, then $f \in \Lambda^{\sigma, M}\left(x_{0}\right)$ if and only if there exist $C>0$ and a unique polynomial $P \in \mathbf{P}[M]$ such that

$$
\|f-P\|_{L^{\infty}\left(x_{0}+B_{j}\right)} \leq C \sigma_{j},
$$

for any $j$ sufficiently large. 
Proof. Let $\left(P_{j}\right)_{j}$ be a sequence of polynomials for which inequality (11) is satisfied and set

$$
P(x)=\sum_{|\beta| \leq M} f_{\beta}\left(x_{0}\right) \frac{\left(x-x_{0}\right)^{\beta}}{\beta !} .
$$

One has

$$
\begin{aligned}
\| P & -P_{j} \|_{L^{\infty}\left(x_{0}+B_{j}\right)} \\
& =\left\|\sum_{|\beta| \leq M}\left(f_{\beta}\left(x_{0}\right)-D^{\beta} P_{j}\left(x_{0}\right)\right) \frac{\left(x-x_{0}\right)^{\beta}}{\beta !}\right\|_{L^{\infty}\left(x_{0}+B_{j}\right)} \\
& \leq \sum_{|\beta| \leq M}\left|f_{\beta}\left(x_{0}\right)-D^{\beta} P_{j}\left(x_{0}\right)\right| 2^{-j|\beta|}
\end{aligned}
$$

Since Lemma 6 implies

$$
\left|f_{\beta}\left(x_{0}\right)-D^{\beta} P_{j}\left(x_{0}\right)\right| \leq C 2^{j|\beta|} \sigma_{j},
$$

for any $j$ sufficiently large, we have

$$
\left\|P-P_{j}\right\|_{L^{\infty}\left(x_{0}+B_{j}\right)} \leq C \sigma_{j} .
$$

This inequality can be used to obtain

$$
\begin{aligned}
\|f-P\|_{L^{\infty}\left(x_{0}+B_{j}\right)} \leq & \left\|f-P_{j}\right\|_{L^{\infty}\left(x_{0}+B_{j}\right)} \\
& +\left\|P_{j}-P\right\|_{L^{\infty}\left(x_{0}+B_{j}\right)} \leq C \sigma_{j},
\end{aligned}
$$

which shows the existence of $P$.

If two polynomials $P, Q \in \mathbf{P}[M]$ satisfy inequality (20), then

$$
\begin{aligned}
\|P-Q\|_{L^{\infty}\left(x_{0}+B_{j}\right)} \leq & \|P-f\|_{L^{\infty}\left(x_{0}+B_{j}\right)} \\
& +\|f-Q\|_{L^{\infty}\left(x_{0}+B_{j}\right)} \leq C \sigma_{j},
\end{aligned}
$$

but if $P \neq Q$,

$$
\|P-Q\|_{L^{\infty}\left(x_{0}+B_{j}\right)} \geq C 2^{-j M},
$$

for any $j$ sufficiently large, so that $2^{j M} \sigma_{j}$ does not tend to zero.

The polynomial $P$ in inequality (20) is the Taylor expansion of $f$, where the derivative is replaced with the Peano derivative.

The spaces $\Lambda^{\sigma, M}\left(x_{0}\right)$ are a generalization of the usual Hölder spaces, defined by (2).

Remark 10. Let $\alpha \in(0, \infty)$; the sequence $\sigma=\left(2^{-j \alpha}\right)_{j \in \mathbf{N}}$ is an admissible sequence with $\underline{s}(\sigma)=\bar{s}(\sigma)=-\alpha, \underline{s}\left(\sigma^{-1}\right)=$ $\bar{s}\left(\sigma^{-1}\right)=\alpha$. Therefore, if $\alpha$ is not a natural number, we have

$$
\Lambda^{\alpha}\left(x_{0}\right)=\Lambda^{\sigma,[\alpha]}\left(x_{0}\right)=\Lambda^{\sigma,\left[\underline{s}\left(\sigma^{-1}\right)\right]}\left(x_{0}\right) .
$$

It is easy to check that the polynomial satisfying (2) is unique if and only if $\alpha \notin \mathbf{N}$. If $\alpha \in \mathbf{N}$, one rather imposes $P \in$ $\mathbf{P}[\alpha-1]$ in order to obtain the uniqueness of the polynomial (one easily verifies that both definitions lead to the same spaces), so that $\Lambda^{\alpha}\left(x_{0}\right)=\Lambda^{\sigma, \underline{s}\left(\sigma^{-1}\right)-1}\left(x_{0}\right)$, with $\sigma_{j}=2^{-j \alpha}$. We will use the modified version in the sequel, ensuring the uniqueness of the polynomial.

The following proposition rigorously expresses the idea that the space $\Lambda^{\sigma, M}\left(x_{0}\right)$ associated with a sequence $\left(\sigma_{j}\right)_{j}$ that decreases faster than $2^{-j M}$ is included in the usual Hölder space $\Lambda^{M}\left(x_{0}\right)$.

Corollary 11. If $\underline{s}\left(\sigma^{-1}\right)>M$, one has $\Lambda^{\sigma, M}\left(x_{0}\right) \subset \Lambda^{M}\left(x_{0}\right)$.

Proof. Let $f \in \Lambda^{\sigma, M}\left(x_{0}\right), P$ be defined as in Theorem 9, that is,

$$
P(x)=\sum_{|\beta| \leq M} f_{\beta}\left(x_{0}\right) \frac{\left(x-x_{0}\right)^{\beta}}{\beta !},
$$

and let us set

$$
Q(x)=\sum_{|\beta| \leq M-1} f_{\beta}\left(x_{0}\right) \frac{\left(x-x_{0}\right)^{\beta}}{\beta !} .
$$

One gets

$$
\begin{aligned}
\|f-Q\|_{L^{\infty}\left(x_{0}+B_{j}\right)} \leq & \|f-P\|_{L^{\infty}\left(x_{0}+B_{j}\right)} \\
& +\|P-Q\|_{L^{\infty}\left(x_{0}+B_{j}\right)} \\
\leq & C \sigma_{j}+C 2^{-j M} \leq C^{\prime} 2^{-j M},
\end{aligned}
$$

since $2^{j M} \sigma_{j}$ tends to zero.

\section{Alternative Definitions of Generalized Hölder Spaces}

Since the uniform spaces $\Lambda^{\sigma}\left(\mathbf{R}^{d}\right)$ can be defined via finite differences or convolutions, one can wonder if such characterizations also hold for the pointwise version of these spaces.

3.1. Characterization in Terms of Finite Differences. As usual, $\Delta_{h}^{n} f$ will stand for the finite difference of order $n$ : given a function $f$ defined on $\mathbf{R}^{d}$ and $x, h \in \mathbf{R}^{d}$,

$$
\begin{aligned}
\Delta_{h}^{1} f(x) & =f(x+h)-f(x), \\
\Delta_{h}^{n+1} f(x) & =\Delta_{h}^{1} \Delta_{h}^{n} f(x),
\end{aligned}
$$

for any $n \in \mathbf{N}$. We also set

$$
B_{h}^{M}\left(x_{0}, j\right)=\left\{x:[x, x+(M+1) h] \subset x_{0}+B_{j}\right\},
$$

where $[x, x+(M+1) h]$ is the line segment with end points $x$ and $x+(M+1) h$. In order to obtain a more general result, we drop the continuity condition of Definition 2 in this section. 
Proposition 12. Let $f \in L_{\text {loc }}^{\infty}\left(\mathbf{R}^{d}\right)$; one has $f \in \Lambda^{\sigma, M}\left(x_{0}\right)$ if and only if there exist $C, J>0$ such that

$$
\sup _{h \in B_{j}}\left\|\Delta_{h}^{M+1} f\right\|_{L^{\infty}\left(B_{h}^{M}\left(x_{0}, j\right)\right)} \leq C \sigma_{j},
$$

for any $j \geq J$.

Proof. The theorem of Whitney (see, e.g., [20]) directly implies that if $f$ satisfies inequality (34), then $f \in \Lambda^{\sigma, M}\left(x_{0}\right)$ : one has

$$
\inf _{P \in \mathbf{P}[M]}\|f-P\|_{L^{\infty}\left(x_{0}+B_{j}\right)} \leq C \sup _{h \in B_{j}}\left\|\Delta_{h}^{M+1} f\right\|_{L^{\infty}\left(B_{h}^{M}\left(x_{0}, j\right)\right)} .
$$

Let us now suppose that $f \in \Lambda^{\sigma, M}\left(x_{0}\right)$ and let $x \in$ $B_{h}^{M}\left(x_{0}, j\right), P \in \mathbf{P}[M]$. One has, using the Fréchet functional equation,

$$
\begin{aligned}
& \left\|\Delta_{h}^{M+1} f\right\|_{L^{\infty}\left(B_{h}^{M}\left(x_{0}, j\right)\right)}=\left\|\Delta_{h}^{M+1}(f-P)\right\|_{L^{\infty}\left(B_{h}^{M}\left(x_{0}, j\right)\right)} \\
& \leq(M+1) !(M+2)\|f-P\|_{L^{\infty}\left(x_{0}+B_{j}\right)} .
\end{aligned}
$$

Now, since there exists a polynomial $P_{j} \in \mathbf{P}[M]$ such that

$$
\left\|f-P_{j}\right\|_{L^{\infty}\left(x_{0}+B_{j}\right)} \leq C \sigma_{j},
$$

for any $j$ sufficiently large, one gets

$$
\sup _{h \in B_{j}}\left\|\Delta_{h}^{M+1} f\right\|_{L^{\infty}\left(B_{h}^{M}\left(x_{0}, j\right)\right)} \leq C \sigma_{j},
$$

for any $j$ sufficiently large.

3.2. Characterization in Terms of Convolutions. Let us denote the space of the infinitely differentiable functions with compact support included in a subset $E$ of $\mathbf{R}^{d}$ by $C_{c}^{\infty}(E)$. In this section, $\rho$ will denote a radial function of $C_{c}^{\infty}(B)$ such that $\rho(x) \in[0,1]$ for any $x \in \mathbf{R}^{d}$ and $\|\rho\|_{1}=1$. Moreover, one sets $\rho_{j}=2^{j d} \rho\left(2^{j}\right.$.), for any $j \in \mathbf{N}$.

In [1], the following result has been obtained.

Lemma 13. Let $N \in \mathbf{N}_{0}$; if $f \in L_{l o c}^{1}\left(\mathbf{R}^{d}\right)$ satisfies

$$
\sup _{k \geq j}\left\|f * \rho_{k}-f\right\|_{L^{\infty}\left(x_{0}+B_{j}\right)} \leq C \sigma_{j},
$$

for $j \geq J$, then, for any multi-index $\beta$ such that $|\beta| \leq N$, one has

$$
\left\|D^{\beta}\left(f * \rho_{j}-f * \rho_{j-1}\right)\right\|_{L^{\infty}\left(x_{0}+B_{j}\right)} \leq C 2^{j N} \sigma_{j},
$$

for $j \geq J+1$.

Using the same ideas as in [1], one gets a similar characterization.

Theorem 14. If $f \in \Lambda^{\sigma, M}\left(x_{0}\right)$, then there exists a function $\Phi \in$ $C_{c}^{\infty}\left(\mathbf{R}^{d}\right)$ such that

$$
\sup _{k \geq j}\left\|f-f * \Phi_{k}\right\|_{L^{\infty}\left(x_{0}+B_{j}\right)} \leq C \sigma_{j},
$$

for any $j$ sufficiently large.
Conversely, if $\sigma \rightarrow 0$, if $f$ is uniformly Hölder and satisfies inequality (41) for a function $\Phi \in C_{c}^{\infty}\left(\mathbf{R}^{d}\right)$, then $f \in \Lambda^{\sigma, M}\left(x_{0}\right)$ for any $M \in \mathbf{N}_{0}$ such that $M+1>\bar{s}\left(\sigma^{-1}\right)$.

Proof. Assume $f \in \Lambda^{\sigma, M}\left(x_{0}\right)$. As in [1] (see also [3]), let us set $\Psi(x)$

$$
=\sum_{j=0}^{m / 2-1}(-1)^{j} \frac{m !}{j !(m-j) !} \frac{1}{(2 j-m)^{d}} \rho\left(\frac{x}{2 j-m}\right),
$$

where $m$ is large enough (larger than $M+1$ ) and $\Phi=$ $\Psi / \int \Psi d x$. Using the same arguments as in [1], one gets

$$
f * \Phi_{k}(x)-f(x)=C \int \Delta_{2^{-k} t}^{m} f(x) \rho(t) d t,
$$

which, due to Proposition 12, leads to inequality (41).

Let us show the converse. Let $\alpha \in(0,1)$ be such that $f \in$ $\Lambda^{\alpha}\left(\mathbf{R}^{d}\right)$ and set, as in [1],

$$
\begin{aligned}
& f_{1}=f * \Phi_{1}, \\
& f_{j}=f *\left(\Phi_{j}-\Phi_{j-1}\right),
\end{aligned}
$$

for $j>1$. Since $f$ is uniformly Hölder, $f$ is uniformly equal to $\sum_{j \geq 1} f_{j}$ on $\mathbf{R}^{d}$ and

$$
\Delta_{h}^{M+1} f=\sum_{j \geq 1} \Delta_{h}^{M+1} f_{j},
$$

uniformly on $\mathbf{R}^{d}$, for any $h \in \mathbf{R}^{d}$. For $j \in \mathbf{N}$, let $n_{0} \in \mathbf{N}_{0}$, $h \in \mathbf{R}^{d}$, and $j_{0} \in \mathbf{N}_{0}$ be such that $M+1<2^{n_{0}},|h| \leq 2^{-\left(j+n_{0}\right)}$, and $2^{-\left(j_{0}+1\right) \alpha} \leq \sigma_{j} \leq 2^{-j_{0} \alpha}$. One has

$$
\begin{aligned}
\left\|\Delta_{h}^{M+1} f\right\|_{L^{\infty}\left(x_{0}+B_{j}\right)} \leq & \sum_{k=1}^{j-1}\left\|\Delta_{h}^{M+1} f_{k}\right\|_{L^{\infty}\left(x_{0}+B_{j}\right)} \\
& +\left\|\sum_{k=j}^{j_{0}} \Delta_{h}^{M+1} f_{k}\right\|_{L^{\infty}\left(x_{0}+B_{j}\right)} \\
& +\sum_{k \geq j_{0}+1}\left\|\Delta_{h}^{M+1} f_{k}\right\|_{L^{\infty}\left(x_{0}+B_{j}\right)},
\end{aligned}
$$

where the second term in the right-hand side only appears if $j \leq j_{0}$.

Using Lemma 13 and the fact that $M+1>\bar{s}\left(\sigma^{-1}\right)$, the mean value theorem allows writing

$$
\begin{aligned}
& \sum_{k=1}^{j-1}\left\|\Delta_{h}^{M+1} f_{k}\right\|_{L^{\infty}\left(x_{0}+B_{j}\right)} \\
& \quad \leq \sum_{k=1}^{j-1} C|h|^{M+1} \sup _{|\beta|=M+1}\left\|D^{\beta} f_{k}\right\|_{L^{\infty}\left(x_{0}+B_{j-1}\right)} \\
& \quad \leq C 2^{-j(M+1)} \sum_{k=1}^{j-1} 2^{k(M+1)} \sigma_{k} \leq C \sigma_{j} .
\end{aligned}
$$


Moreover,

$$
\begin{aligned}
& \left\|\sum_{k=j}^{j_{0}} \Delta_{h}^{M+1} f_{k}\right\|_{L^{\infty}\left(x_{0}+B_{j}\right)} \\
& \quad=\left\|\Delta_{h}^{M+1}\left(f * \Phi_{j_{0}}-f * \Phi_{j-1}\right)\right\|_{L^{\infty}\left(x_{0}+B_{j}\right)} \leq C \| f \\
& \quad * \Phi_{j_{0}}-f * \Phi_{j-1} \|_{L^{\infty}\left(x_{0}+B_{j-1}\right)} \\
& \quad \leq C\left(\left\|f * \Phi_{j_{0}}-f\right\|_{L^{\infty}\left(x_{0}+B_{j-1}\right)}\right. \\
& \left.\quad+\left\|f-f * \Phi_{j-1}\right\|_{L^{\infty}\left(x_{0}+B_{j-1}\right)}\right) \leq C \sigma_{j-1}+C \sigma_{j-1} \\
& \quad \leq C \sigma_{j} .
\end{aligned}
$$

Finally,

$$
\begin{aligned}
& \sum_{k \geq j_{0}+1}\left\|\Delta_{h}^{M+1} f_{k}\right\|_{L^{\infty}\left(x_{0}+B_{j}\right)} \leq C \sum_{k \geq j_{0}+1}\left\|f_{k}\right\|_{L^{\infty}\left(\mathbf{R}^{d}\right)} \\
& \leq C \sum_{k \geq j_{0}+1} 2^{-k \alpha} \leq 2^{-j_{0} \alpha} \leq C \sigma_{j} .
\end{aligned}
$$

One then has

$$
\begin{aligned}
& \sup _{h \in B_{j+n_{0}}}\left\|\Delta_{h}^{M+1} f\right\|_{L^{\infty}\left(x_{0}+B_{j+n_{0}}\right)} \leq \sup _{h \in B_{j+n_{0}}}\left\|\Delta_{h}^{M+1} f\right\|_{L^{\infty}\left(x_{0}+B_{j}\right)} \\
& \quad \leq C \sigma_{j} \leq C \sigma_{j+n_{0}},
\end{aligned}
$$

as wanted, in view of Proposition 12.

\section{Generalized Pointwise Hölder Spaces and Wavelets}

The usual Hölder spaces can "nearly" be characterized in terms of wavelets [12]: for the sufficiency of the condition, the function has to be uniformly Hölder and a logarithmic correction appears. We show here that such a result still holds in the generalized case.

4.1. Definitions. Let us briefly recall some definitions and notations (for more precisions, see, e.g., [21-23]). Under some general assumptions, there exist a real-valued function $\phi$ and $2^{d}-1$ real-valued functions $\left(\psi^{(i)}\right)_{1 \leq i<2^{d}}$ defined on $\mathbf{R}^{d}$, called wavelets, such that

$$
\begin{aligned}
& \left\{\phi(\cdot-k): k \in \mathbf{Z}^{d}\right\} \\
& \quad \cup\left\{\psi^{(i)}\left(2^{j} \cdot-k\right): 1 \leq i<2^{d}, k \in \mathbf{Z}^{d}, j \in \mathbf{N}_{0}\right\},
\end{aligned}
$$

form an orthogonal basis of $L^{2}\left(\mathbf{R}^{d}\right)$. Any function $f \in L^{2}\left(\mathbf{R}^{d}\right)$ can be decomposed as follows:

$$
\begin{aligned}
f(x)= & \sum_{k \in \mathbf{Z}^{d}} C_{k} \phi(x-k) \\
& +\sum_{j=0}^{+\infty} \sum_{k \in \mathbf{Z}^{d}} \sum_{1 \leq i<2^{d}} c_{j, k}^{(i)} \psi^{(i)}\left(2^{j} x-k\right),
\end{aligned}
$$

where

$$
\begin{aligned}
c_{j, k}^{(i)} & =2^{d j} \int_{\mathbf{R}^{d}} f(x) \psi^{(i)}\left(2^{j} x-k\right) d x, \\
C_{k} & =\int_{\mathbf{R}^{d}} f(x) \phi(x-k) d x .
\end{aligned}
$$

Let us remark that we do not choose the $L^{2}\left(\mathbf{R}^{d}\right)$ normalization for the wavelets, but rather an $L^{\infty}$ normalization, which is better fitted to the study of the Hölderian regularity. Hereafter, the wavelets are always supposed to belong to $C^{n}\left(\mathbf{R}^{d}\right)$ with $n>M$, and the functions $\left(D^{s} \phi\right)_{|s| \leq \gamma},\left(D^{s} \psi^{(i)}\right)_{|s| \leq \gamma}$ are assumed to have fast decay (where $\gamma$ is a sufficiently large number, i.e., strictly greater than $M$ ).

A dyadic cube of scale $j$ is a cube of the form

$$
\lambda=\left[\frac{k_{1}}{2^{j}}, \frac{k_{1}+1}{2^{j}}\right) \times \cdots \times\left[\frac{k_{d}}{2^{j}}, \frac{k_{d}+1}{2^{j}}\right),
$$

where $k=\left(k_{1}, \ldots, k_{d}\right) \in \mathbf{Z}^{d}$. From now on, wavelets and wavelet coefficients will be indexed with dyadic cubes $\lambda$. Since $i$ takes $2^{d}-1$ values, we can assume that it takes values in $\{0,1\}^{d}-(0, \ldots, 0)$; we will use the following notations:

$$
\begin{aligned}
& \text { (i) } \lambda=\lambda(i, j, k)=k / 2^{j}+i / 2^{j+1}+\left[0,1 / 2^{j+1}\right)^{d}, \\
& \text { (ii) } c_{\lambda}=c_{j, k}^{(i)}, \\
& \text { (iii) } \psi_{\lambda}=\psi_{j, k}^{(i)}=\psi^{(i)}\left(2^{j} \cdot-k\right) .
\end{aligned}
$$

The pointwise Hölderian regularity of a function is closely related to the decay rate of its wavelet leaders.

Definition 15. The wavelet leaders are defined by

$$
d_{\lambda}=\sup _{\lambda^{\prime} \subset \lambda}\left|c_{\lambda^{\prime}}\right|
$$

Two dyadic cubes $\lambda$ and $\lambda^{\prime}$ are adjacent if they are at the same scale and if $\operatorname{dist}\left(\lambda, \lambda^{\prime}\right)=0$. We denote by $3 \lambda$ the set of the $3^{d}$ dyadic cubes adjacent to $\lambda$ and by $\lambda_{j}\left(x_{0}\right)$ the dyadic cube of side length $2^{-j}$ containing $x_{0}$; we then set

$$
d_{j}\left(x_{0}\right)=\sup _{\lambda \subset 3 \lambda_{j}\left(x_{0}\right)} d_{\lambda} .
$$

4.2. Result. From now on, we will suppose that the wavelets are compactly supported (such wavelets are constructed in [24]) and $j_{0}$ will stand for a natural number such that the support of $\psi^{(i)}$ is included in $2^{j_{0}} B$, for any $i \in\left\{1, \ldots, 2^{d}-1\right\}$.

Theorem 16. If $f \in \Lambda^{\sigma, M}\left(x_{0}\right)$, then there exist $C>0$ and $J \in \mathbf{N}_{0}$ such that

$$
d_{j}\left(x_{0}\right) \leq C \sigma_{j}
$$

for any $j \geq J$.

Conversely, let $f$ be a uniformly Hölder function; if inequality (57) is satisfied for an admissible sequence $\sigma$ that tends to zero, then $f \in \Lambda^{\tau, M}\left(x_{0}\right)$, where $\tau$ is the admissible sequence defined by $\tau_{j}=\sigma_{j}\left|\log _{2} \sigma_{j}\right|$ and $M \in \mathbf{N}_{0}$ is any number satisfying $M+1>\bar{s}\left(\sigma^{-1}\right)$. 
Proof. In what follows, $P_{j-k_{0}} \in \mathbf{P}[M]$ is according to (11). If $f \in \Lambda^{\sigma, M}\left(x_{0}\right)$, let $k_{0} \in \mathbf{N}_{0}$ be such that $2^{j_{0}+1}+4 d \leq 2^{k_{0}}$. For $j \geq k_{0}+1$ and $\lambda=\lambda\left(i, j^{\prime}, k^{\prime}\right) \subset 3 \lambda_{j}\left(x_{0}\right)$, one has

$$
\begin{aligned}
\left|c_{\lambda}\right| & =\left|2^{d j^{\prime}} \int f(x) \psi_{\lambda}(x) d x\right| \\
& =\left|2^{d j^{\prime}} \int\left(f(x)-P_{j-k_{0}}(x)\right) \psi_{\lambda}(x) d x\right| \\
& =\left|2^{d j^{\prime}} \int_{k^{\prime} / 2^{j^{\prime}+B_{j^{\prime}}-j_{0}}}\left(f(x)-P_{j-k_{0}}(x)\right) \psi_{\lambda}(x) d x\right| \\
& \leq 2^{d j^{\prime}} \int_{x_{0}+B_{j-k_{0}}}\left|f(x)-P_{j-k_{0}}(x)\right|\left|\psi_{\lambda}(x)\right| d x \\
& \leq C 2^{d j^{\prime}} \sigma_{j-k_{0}} \int\left|\psi_{\lambda}(x)\right| d x \leq C \sigma_{j},
\end{aligned}
$$

which is the desired result.

Now, let us suppose that inequality (57) is satisfied for a function $f \in \Lambda^{\varepsilon}\left(\mathbf{R}^{d}\right)$ and an admissible sequence $\sigma$ tending to 0 . Let us set

$$
\begin{aligned}
f_{-1} & =\sum_{k} C_{k} \phi(\cdot-k), \\
f_{j} & =\sum_{i, k} c_{\lambda} \psi_{\lambda},
\end{aligned}
$$

for $j \in \mathbf{N}_{0}$. In [2], it has been shown that these functions have the same regularity as the wavelets and that $f$ is uniformly equal to $\sum_{j \geq-1} f_{j}$. Let us define

$$
P_{J}(x)=\sum_{|\beta| \leq M} \frac{\left(x-x_{0}\right)^{\beta}}{\beta !} \sum_{j=-1}^{J} D^{\beta} f_{j}\left(x_{0}\right),
$$

and let us choose $n_{d} \in \mathbf{N}$ such that $R>2^{-j}$ and $k / 2^{j} \in x+R B$ $\left(x \in \mathbf{R}^{d}\right)$ implies

$$
\frac{k}{2^{j}}+\frac{i}{2^{j+1}}+\left[0, \frac{1}{2^{j}}\right)^{d} \subset x+2^{n_{d}} R B
$$

Let us also choose $m_{d} \in \mathbf{N}$ such that any ball $x+B_{j}\left(x \in \mathbf{R}^{d}\right.$, $j \in \mathbf{N}_{0}$ ) is included in a dyadic cube of length $2^{m_{d^{-j}}}$. If $J^{\prime}$ is such that $\sigma_{j}<1$ for any $j \geq J^{\prime}$, we finally choose $J$ such that $J \geq \sup \left\{J^{\prime}, j_{0}+n_{d}+m_{d}+1\right\}$. One has

$$
\begin{aligned}
\| f- & P_{J} \|_{L^{\infty}\left(x_{0}+B_{J}\right)} \\
\leq & \sum_{j=-1}^{J}\left\|f_{j}(x)-\sum_{|\beta| \leq M} \frac{\left(x-x_{0}\right)^{\beta}}{\beta !} D^{\beta} f_{j}\left(x_{0}\right)\right\|_{L^{\infty}\left(x_{0}+B_{J}\right)} \\
& +\sum_{j \geq J+1}\left\|f_{j}\right\|_{L^{\infty}\left(x_{0}+B_{J}\right)} .
\end{aligned}
$$

Let us look at the first term of the right-hand side. Let $j \leq J$; using the Taylor expansion, one gets

$$
\begin{gathered}
\left\|f_{j}(x)-\sum_{|\beta| \leq M} \frac{\left(x-x_{0}\right)^{\beta}}{\beta !} D^{\beta} f_{j}\left(x_{0}\right)\right\|_{L^{\infty}\left(x_{0}+B_{J}\right)} \\
\leq C 2^{-J(M+1)} \sup _{|\beta|=M+1}\left\|D^{\beta} f_{j}\right\|_{L^{\infty}\left(x_{0}+B_{J}\right)} .
\end{gathered}
$$

If $\beta$ satisfies $|\beta|=M+1$, we have, for any $x \in x_{0}+B_{J}$,

$$
\begin{aligned}
\left|D^{\beta} f_{j}(x)\right| & \leq \sum_{i, k} 2^{j(M+1)}\left|c_{\lambda}\right|\left|D^{\beta} \psi_{\lambda}(x)\right| \\
& =\sum_{i} \sum_{k 2^{-j} \in x+B_{j-j_{0}}} 2^{j(M+1)}\left|c_{\lambda}\right|\left|D^{\beta} \psi_{\lambda}(x)\right| .
\end{aligned}
$$

Each coefficient $c_{\lambda}$ in the last sum is such that $\lambda \subset x+B_{j-j_{0}-n_{d}}$. Therefore, if $j \geq j_{0}+n_{d}+m_{d}+1$, then

$$
\left|c_{\lambda}\right| \leq C \sigma_{j-j_{0}-n_{d}-m_{d}-1} .
$$

Otherwise, since $f$ is uniformly Hölder, $\left|c_{\lambda}\right| \leq C \leq C \sigma_{j}$. Therefore,

$$
\left\|D^{\beta} f_{j}\right\|_{L^{\infty}\left(x_{0}+B_{J}\right)} \leq C 2^{j(M+1)} \sigma_{j},
$$

for any $j \leq J$, which implies

$$
\begin{aligned}
& \sum_{j=-1}^{J}\left\|f_{j}(x)-\sum_{|\beta| \leq M} \frac{\left(x-x_{0}\right)^{\beta}}{\beta !} D^{\beta} f_{j}\left(x_{0}\right)\right\|_{L^{\infty}\left(x_{0}+B_{J}\right)} \\
& \quad \leq C 2^{-J(M+1)} \sum_{j=-1}^{J} 2^{j(M+1)} \sigma_{j} \leq C \sigma_{J} .
\end{aligned}
$$

For the second term in the right-hand side of (62), let us define $J_{1} \in \mathbf{N}$ as the number such that $2^{-\varepsilon J_{1}} \leq \sigma_{J}<2^{-\varepsilon\left(J_{1}-1\right)}$ and decompose the sum as follows:

$$
\begin{aligned}
\sum_{j \geq J+1}\left\|f_{j}\right\|_{L^{\infty}\left(x_{0}+B_{J}\right)}= & \sum_{j \geq J_{1}+1}\left\|f_{j}\right\|_{L^{\infty}\left(x_{0}+B_{J}\right)} \\
& +\sum_{j=J+1}^{J_{1}}\left\|f_{j}\right\|_{L^{\infty}\left(x_{0}+B_{J}\right)} .
\end{aligned}
$$

We have

$$
\begin{aligned}
\sum_{j \geq J_{1}+1}\left\|f_{j}\right\|_{L^{\infty}\left(x_{0}+B_{J}\right)} & \leq \sum_{j \geq J_{1}+1}\left\|f_{j}\right\|_{L^{\infty}\left(\mathbf{R}^{d}\right)} \leq C \sum_{j \geq J_{1}+1} 2^{-\varepsilon j} \\
& \leq C 2^{-\varepsilon I_{1}} \leq C \sigma_{J} .
\end{aligned}
$$

Now, for $j \in\left\{J+1, \ldots, J_{1}\right\}$ and $x \in x_{0}+B_{J}$, one has

$$
\left|f_{j}(x)\right| \leq \sum_{i} \sum_{k 2^{-j} \in x+B_{j-j_{0}}}\left|c_{\lambda} \psi_{\lambda}(x)\right| .
$$


If $j \geq J+j_{0}+n_{d}$, the wavelet coefficients $c_{\lambda}$ in the last sum are such that

$$
\lambda \subset x+B_{j-j_{0}-n_{d}} \subset x_{0}+B_{J-1},
$$

and therefore

$$
\left|c_{\lambda}\right| \leq C \sigma_{J-m_{d}-1} \leq C \sigma_{J} .
$$

In the other case,

$$
\lambda \subset x+B_{j-j_{0}-n_{d}} \subset x_{0}+B_{j-j_{0}-n_{d}-1},
$$

and thus

$$
\left|c_{\lambda}\right| \leq C \sigma_{j-j_{0}-n_{d}-m_{d}-1} \leq C \sigma_{j} \leq C \sigma_{J} .
$$

These inequalities lead to

$$
\sum_{j=J+1}^{J_{1}}\left\|f_{j}\right\|_{L^{\infty}\left(x_{0}+B_{J}\right)} \leq C J_{1} \sigma_{J} \leq C\left|\log _{2}\left(\sigma_{J}\right)\right| \sigma_{J} .
$$

Putting all these inequalities together, one gets

$$
\left\|f-P_{J}\right\|_{L^{\infty}\left(x_{0}+B_{J}\right)} \leq C\left|\log _{2}\left(\sigma_{J}\right)\right| \sigma_{J},
$$

as desired.

The converse part of the previous theorem requires a uniform regularity condition. As shown in [25], a stronger condition than continuity is necessary in the usual case (see also [26], where similar results are obtained (in the usual case) with a Besov regularity assumption). Similarly, the logarithmic correction is best possible in the usual case [25].

\section{A Generalized Definition of the Hölder Exponent}

The usual Hölder spaces are embedded: $\alpha<\beta$ implies $\Lambda^{\beta}\left(x_{0}\right) \subset \Lambda^{\alpha}\left(x_{0}\right)$. A notion of regularity for a function $f \in L_{\text {loc }}^{\infty}\left(\mathbf{R}^{d}\right)$ at $x_{0}$ can thus be given by the so-called Hölder exponent:

$$
h_{f}\left(x_{0}\right)=\sup \left\{\alpha>0: f \in \Lambda^{\alpha}\left(x_{0}\right)\right\} .
$$

To do so in the generalized case, one needs some conditions under which $\Lambda^{\sigma, M}\left(x_{0}\right) \subset \Lambda^{\sigma^{\prime}, M^{\prime}}\left(x_{0}\right)$.

This generalized exponent naturally leads to the definition of an alternative multifractal formalism, yet similar to the one developed in [12], where, for example, logarithmic corrections can appear.

5.1. A Trivial Illustration. The classical version of Theorem 16 theoretically allows estimating the Hölder regularity at a given point $x_{0}$ by looking at the behavior of $d_{j}\left(x_{0}\right)$ versus the scales $j$ [12]. This notion of regularity is given by the Hölder exponent $h_{f}\left(x_{0}\right)$, defined by equality (77). Following the standard wavelet characterization [12], one should have

$$
\log _{2} d_{j}\left(x_{0}\right) \sim h_{f}\left(x_{0}\right) j+C,
$$

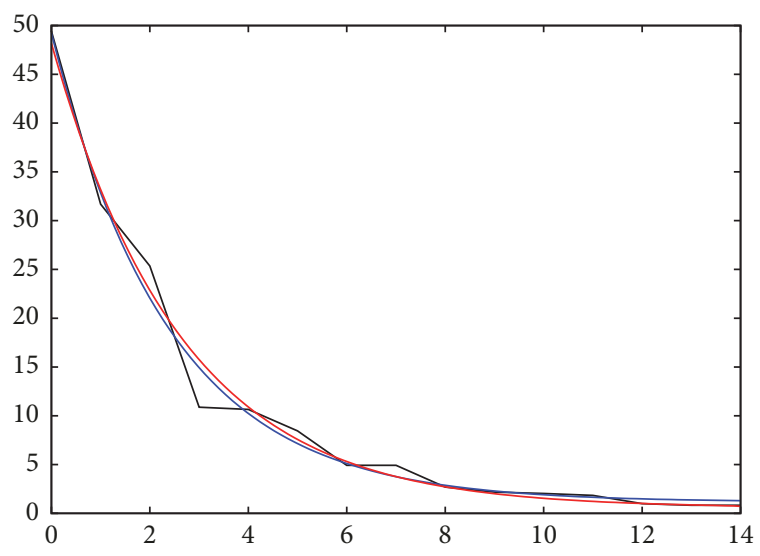

FIGURE 1: The function $j \mapsto d_{j}\left(x_{0}\right)$ in black, with the standard curve $C 2^{-j h}$ (blue) and the logarithmically corrected curve (using equality (80)) (red) obtained with the Levenberg-Marquardt algorithm.

and a log-log plot can be used to estimate the slope $h_{0}$. This method is simpler than directly fitting the curve

$$
\gamma_{C, h}(t)=C \omega^{(h)}\left(2^{-j}\right),
$$

with $\omega^{(h)}(r)=r^{h}$ to the function $j \mapsto d_{j}\left(x_{0}\right)$, since in this latter case one has to estimate two parameters $(C$ and $h)$ to retain only one (namely, $h$, which is the estimation of $h_{f}\left(x_{0}\right)$ ). However, this approach allows fine-tuning the computation of $h_{0}$ using Theorem 16. In the case of a Brownian motion, for example, having the law of the iterated logarithm in mind $[14,15]$, one should rather choose

$$
\omega^{(h)}(r)=(r \log |\log r|)^{h},
$$

in the definition of $\gamma_{C, h}$. Fitting $d_{j}\left(x_{0}\right)$ with different definitions of $\omega^{(h)}$ should help to discern between specific models. In the case proposed here, it could support the detection of the presence of a logarithmic correction, which could be the signature of a Brownian motion.

As an illustration, the wavelet leader of a Brownian motion $j \mapsto d_{j}\left(x_{0}\right)$ for some $x_{0}$ (the middle was arbitrarily chosen) is represented in Figure 1. When trying to fit the curve $C 2^{-j h}$ to $d_{j}\left(x_{0}\right)$ using the Levenberg-Marquardt algorithm [27], one gets $h_{f}\left(x_{0}\right)=0.554$ (see Figure 1). The same computation with the logarithmic correction (using Definition (80)) gives $h_{f}\left(x_{0}\right)=0.495$, which is closer to the expected value $1 / 2$. Computing the distance, for each point $x_{0}$ of the signal ( $2^{20}$ points), between the estimated Hölder exponent $h_{f}\left(x_{0}\right)$ and the expected value $1 / 2$ gives rise to the boxplot represented in Figure 2 .

Of course, this application is only a simple illustrative example; more sophisticated work is required to show the effectiveness of this approach and is out of the scope of this work (see [28]). From a numerical point of view, the estimation of $h_{f}\left(x_{0}\right)$ is unstable (in particular, the detection of fast and slow points in the Brownian motion seems to be a difficult task, if not insufferable); this is why one rather tries to estimate the size of the set of points sharing the same Hölder 


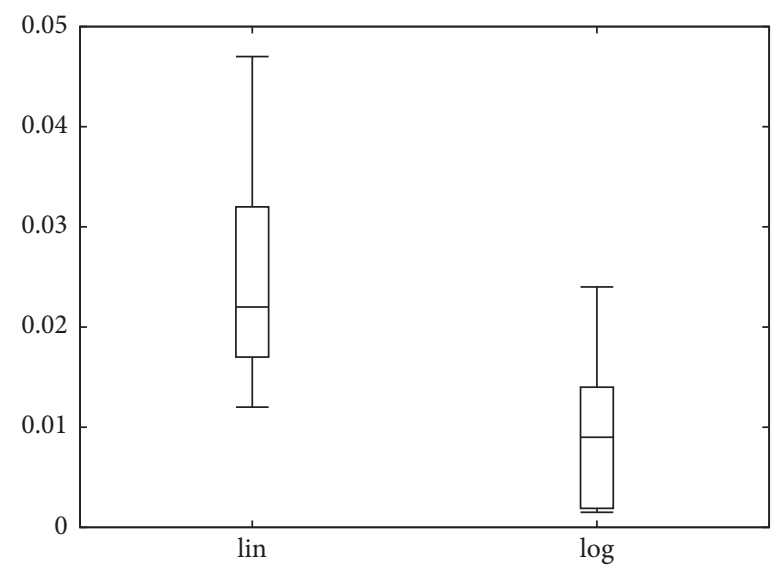

FIGURE 2: Boxplot of the distance between $1 / 2$ and the estimated Hölder exponent. The left boxplot corresponds to the usual method and the other corresponds to a method involving the logarithmic correction.

exponent. This is the purpose of the multifractal formalism for functions (see, e.g., [12]), which will not be recalled here.

5.2. Preliminary Results. We first need some technical easy results. From now on, if $f \in \Lambda^{\sigma, M}\left(x_{0}\right),\left(P_{j}\right)_{j}$ will stand for the sequence of polynomials of $\mathbf{P}[M]$ corresponding to the definition. We will write

$$
\begin{aligned}
P_{j}(x) & =\sum_{|\beta| \leq M} a_{j}^{(\beta)} x^{\beta}, \\
Q_{j}(x) & =\sum_{|\beta| \leq M-1} a_{j}^{(\beta)} x^{\beta} .
\end{aligned}
$$

Lemma 17. Let $f \in \Lambda^{\sigma, M}\left(x_{0}\right)$; one has

$$
\begin{aligned}
& \sup _{|\beta|=M}\left|a_{j}^{(\beta)}\right| \leq C\left(\sum_{k=1}^{j-1}\left(2^{M} \bar{\Theta}_{1}\right)^{k}+1\right), \\
& \sup _{|\beta|=M}\left|a_{j}^{(\beta)}\right| \leq C\left(\sigma_{j} \underline{\Theta}_{1}^{-j} \sum_{k=1}^{j-1}\left(2^{M} \underline{\Theta}_{1}\right)^{k}+1\right) .
\end{aligned}
$$

Proof. Using the Markov inequality (13), we get

$$
\begin{aligned}
& \left\|D^{\beta}\left(P_{j}-P_{j+1}\right)\right\|_{L^{\infty}\left(x_{0}+B_{j+1}\right)} \\
& \leq C 2^{j M}\left\|P_{j}-P_{j+1}\right\|_{L^{\infty}\left(x_{0}+B_{j+1}\right)} \\
& \leq C 2^{j M}\left(\left\|P_{j}-f\right\|_{L^{\infty}\left(x_{0}+B_{j}\right)}+\left\|f-P_{j+1}\right\|_{L^{\infty}\left(x_{0}+B_{j+1}\right)}\right) \\
& \leq C 2^{j M} \sigma_{j},
\end{aligned}
$$

for any $\beta$ such that $|\beta| \leq M$ and $j$ sufficiently large. Therefore, we have

$$
\begin{aligned}
& \left\|D^{\beta}\left(P_{1}-P_{j}\right)\right\|_{L^{\infty}\left(x_{0}+B_{j}\right)} \\
& \leq \sum_{k=1}^{j-1}\left\|D^{\beta}\left(P_{k}-P_{k+1}\right)\right\|_{L^{\infty}\left(x_{0}+B_{j}\right)} \\
& \leq \sum_{k=1}^{j-1}\left\|D^{\beta}\left(P_{k}-P_{k+1}\right)\right\|_{L^{\infty}\left(x_{0}+B_{k+1}\right)} \leq C \sum_{k=1}^{j-1} 2^{k M} \sigma_{k} \\
& \leq C \sum_{k=1}^{j-1}\left(2^{M} \bar{\Theta}_{1}\right)^{k},
\end{aligned}
$$

for any $j$.

Now, let $\beta$ be a multi-index such that $|\beta|=M$; inequality (82) follows from

$$
\left\|D^{\beta}\left(P_{1}-P_{j}\right)\right\|_{L^{\infty}\left(x_{0}+B_{j}\right)} \geq C\left(\left|a_{j}^{(\beta)}\right|-\left|a_{1}^{(\beta)}\right|\right),
$$

while inequality (83) can be obtained in the same way, using

$$
\begin{gathered}
\left\|D^{\beta}\left(P_{1}-P_{j}\right)\right\|_{L^{\infty}\left(x_{0}+B_{j}\right)} \leq C \sum_{k=1}^{j-1} 2^{k M} \sigma_{k} \\
\leq C \sigma_{j} \underline{\Theta}_{1}^{-j} \sum_{k=1}^{j-1}\left(2^{M} \underline{\Theta}_{1}\right)^{k},
\end{gathered}
$$

valid for any $j$.

Corollary 18. Let $f \in \Lambda^{\sigma, M}\left(x_{0}\right)$; one has the following inequalities:

(i) if $2^{M} \bar{\Theta}_{1}<1$,

$$
\left\|f-Q_{j}\right\|_{L^{\infty}\left(x_{0}+B_{j}\right)} \leq C\left(\sigma_{j}+2^{-j M}\right),
$$

(ii) if $2^{M} \bar{\Theta}_{1}>1$,

$$
\left\|f-Q_{j}\right\|_{L^{\infty}\left(x_{0}+B_{j}\right)} \leq C\left(\sigma_{j}+\bar{\Theta}_{1}^{j}\right),
$$

(iii) if $2^{M} \bar{\Theta}_{1}=1$,

$$
\left\|f-Q_{j}\right\|_{L^{\infty}\left(x_{0}+B_{j}\right)} \leq C\left(\sigma_{j}+2^{-j M} j\right) .
$$

Corollary 19. Let $f \in \Lambda^{\sigma, M}\left(x_{0}\right)$; one has the following inequalities:

(i) if $2^{M} \underline{\Theta}_{1}<1$,

$$
\left\|f-Q_{j}\right\|_{L^{\infty}\left(x_{0}+B_{j}\right)} \leq C\left(\sigma_{j}\left(2^{M} \underline{\Theta}_{1}\right)^{-j}+2^{-j M}\right),
$$

(ii) if $2^{M} \underline{\Theta}_{1}>1$,

$$
\left\|f-Q_{j}\right\|_{L^{\infty}\left(x_{0}+B_{j}\right)} \leq C\left(\sigma_{j}+2^{-j M}\right),
$$

(iii) if $2^{M} \underline{\Theta}_{1}=1$,

$$
\left\|f-Q_{j}\right\|_{L^{\infty}\left(x_{0}+B_{j}\right)} \leq C\left(\sigma_{j} j+2^{-j M}\right) .
$$


5.3. Definitions. Before introducing the definition of a generalized Hölder exponent, we must first consider embedded spaces of type $\Lambda^{\sigma, M}\left(x_{0}\right)$. Once the definitions are given, we provide sufficient conditions for generalized Hölder spaces to be embedded.

Definition 20. If, for any $\alpha>0, \sigma^{(\alpha)}$ is an admissible sequence, the application

$$
\sigma^{(\cdot)}: \alpha>0 \longmapsto \sigma^{(\alpha)}
$$

is called a family of admissible sequences.

Definition 21. Let $x_{0} \in \mathbf{R}^{d}$; a family $\sigma^{(\cdot)}$ of admissible sequences is decreasing for $x_{0}$ if $\alpha>\beta$ implies $\Lambda^{\sigma^{(\alpha)}{ }^{,}[\alpha]}\left(x_{0}\right) \subset$ $\Lambda^{\sigma^{(\beta)},[\beta]}\left(x_{0}\right)$.

Definition 22. Let $\sigma^{(\cdot)}$ be a decreasing family of admissible sequences for $x_{0}$; if $f \in L_{\mathrm{loc}}^{\infty}\left(\mathbf{R}^{d}\right)$, then the Hölder exponent of $f$ at $x_{0}$ for the family $\sigma^{(\cdot)}$ is given by

$$
h_{f}^{\sigma^{(\cdot)}}\left(x_{0}\right)=\sup \left\{\alpha>0: f \in \Lambda^{\sigma^{(\alpha)},[\alpha]}\left(x_{0}\right)\right\} \text {. }
$$

The following proposition is a simple corollary of the results obtained in the previous section; it helps to check if a family of admissible sequences is decreasing. If $\sigma^{(\cdot)}$ is a family of admissible sequences, we set

$$
\begin{gathered}
\underline{\Theta}_{j}^{(\alpha)}=\inf _{k \in \mathbf{N}} \frac{\sigma_{j+k}^{(\alpha)}}{\sigma_{k}^{(\alpha)}}, \\
\bar{\Theta}_{j}^{(\alpha)}=\sup _{k \in \mathbf{N}} \frac{\sigma_{j+k}^{(\alpha)}}{\sigma_{k}^{(\alpha)}} .
\end{gathered}
$$

Proposition 23. Let $\sigma^{(\cdot)}$ be a family of admissible sequences and $x_{0} \in \mathbf{R}^{d} ; \sigma^{(\cdot)}$ is decreasing for $x_{0}$ if it satisfies the following two conditions:

(1) if $m \leq \alpha<\beta<m+1$, with $m \in \mathbf{N}_{0}$, then there exist $C, J>0$ such that

$$
\sigma_{j}^{(\beta)} \leq C \sigma_{j}^{(\alpha)}
$$

for any $j \geq J$,

(2) for any $m \in \mathbf{N}$, at least one of the following two conditions is satisfied:

(a) there exists $\varepsilon_{0}>0$ such that, for any $\varepsilon \in\left(0, \varepsilon_{0}\right)$, there exist $C, J>0$ for which $\sigma_{j}^{(m)} \leq C \sigma_{j}^{(m-\varepsilon)}$ and

(i) if $1<2^{m} \bar{\Theta}_{1}^{(m)}$, then $\left(\bar{\Theta}_{1}^{(m)}\right)^{j} \leq C \sigma_{j}^{(m-\varepsilon)}$,

(ii) if $1>2^{m} \bar{\Theta}_{1}^{(m)}$, then $2^{-j m} \leq C \sigma_{j}^{(m-\varepsilon)}$,

(iii) if $1=2^{m} \bar{\Theta}_{1}^{(m)}$, then $j 2^{-j m} \leq C \sigma_{j}^{(m-\varepsilon)}$, for any $j \geq J$, (b) there exists $\varepsilon_{0}>0$ such that, for any $\varepsilon \in\left(0, \varepsilon_{0}\right)$, there exist $C, J>0$ for which $2^{-j m} \leq C \sigma_{j}^{(m-\varepsilon)}$ and

(i) if $1<2^{m} \underline{\Theta}_{1}^{(m)}$, then $\sigma_{j}^{(m)} \leq C \sigma_{j}^{(m-\varepsilon)}$,

(ii) if $1>2^{m} \underline{\Theta}_{1}^{(m)}$, then $\sigma_{j}^{(m)}\left(2^{m} \underline{\Theta}_{1}^{(m)}\right)^{-j} \leq$ $C \sigma_{j}^{(m-\varepsilon)}$,

(iii) if $1=2^{m} \underline{\Theta}_{1}^{(m)}$, then $j \sigma_{j}^{(m)} \leq C \sigma_{j}^{(m-\varepsilon)}$,

for any $j \geq J$.

This result is similar to the one obtained in [1] (under the hypothesis of Proposition 23, one gets a decreasing family of admissible sequences for the uniform case), but the proof given for these generalized uniform Hölder spaces cannot be adapted for the pointwise case.

Remark 24. Since the family of admissible sequences $\sigma^{(\cdot)}$ defined by $\sigma_{j}^{(\alpha)}=2^{-j \alpha}$ satisfies the conditions of Proposition 23 , this result implies the classical inclusion $\Lambda^{\beta}\left(x_{0}\right) \subset$ $\Lambda^{\alpha}\left(x_{0}\right)$, for any $\alpha, \beta$ satisfying $\alpha<\beta$.

Let us consider the following example to grasp the practicality of Proposition 23.

Example 25. Let $g: \alpha>0 \mapsto g(\alpha)$ be a positive function and set

$$
\sigma_{j}^{(\alpha)}=2^{-j \alpha} j^{g(\alpha)}
$$

One directly checks that condition (2)(b) is satisfied, so that $\sigma^{(\cdot)}$ is a decreasing family of admissible sequences.

Both conditions can be used to obtain decreasing families of admissible sequences; here is an example where the first condition is used.

Example 26. Let

$$
g(\alpha)= \begin{cases}-1 & \text { if } \alpha \in \mathbf{N} \\ 0 & \text { otherwise }\end{cases}
$$

If $\sigma^{(\cdot)}$ is defined using identity (98), condition (2)(a) is satisfied so that this sequence is also a decreasing family of admissible sequences.

\section{Data Availability}

No data were used to support this study.

\section{Disclosure}

Some of the results of this manuscript were presented in the Belgium Congress of Mathematics.

\section{Conflicts of Interest}

The authors declare that they have no conflicts of interest. 


\section{References}

[1] D. Kreit and S. Nicolay, "Some characterizations of generalized Hölder spaces," Mathematische Nachrichten, vol. 285, no. 17-18, pp. 2157-2172, 2012.

[2] D. Kreit and S. Nicolay, "Characterizations of the elements of generalized Hölder-Zygmund spaces by means of their representation," Journal of Approximation Theory, vol. 172, pp. 23-36, 2013.

[3] S. G. Krantz, "Lipschitz spaces, smoothness of functions, and approximation theory," Exposition. Math, vol. 1, no. 3, pp. 193260, 1983.

[4] M. Clausel and S. Nicolay, "Some prevalent results about strongly monoHölder functions," Nonlinearity, vol. 23, no. 9, pp. 2101-2116, 2010.

[5] A. M. Caetano and S. D. Moura, "Local growth envelopes of spaces of generalized smoothness: The critical case," Journal of Inequalities and Applications, vol. 7, no. 4, pp. 573-606, 2004.

[6] W. Farkas, "Function spaces of generalised smoothness and pseudo-differential operators associated to a continuous negative definite function," in Habilitation Thesis, 2002.

[7] A. Almeida, "Wavelet bases in generalized Besov spaces," Journal of Mathematical Analysis and Applications, vol. 304, no. 1, pp. 198-211, 2005.

[8] W. Farkas and H.-G. Leopold, "Characterisations of function spaces of generalised smoothness," Annali di Matematica Pura ed Applicata, vol. 185, no. 1, pp. 1-62, 2006.

[9] T. Kühn, H.-G. Leopold, W. Sickel, and L. Skrzypczak, "Entropy numbers of embeddings of weighted besov spaces. II," Proceedings of the Edinburgh Mathematical Society, vol. 49, no. 2, pp. 331-359, 2006.

[10] S. D. Moura, "On some characterizations of Besov spaces of generalized smoothness," Mathematische Nachrichten, vol. 280, no. 9-10, pp. 1190-1199, 2007.

[11] S. Jaffard and Y. Meyer, "Wavelet methods for pointwise regularity and local oscillations of functions," Memoirs of the American Mathematical Society, no. 587, pp. 1-108, 1996.

[12] S. Jaffard, "Wavelet techniques in multifractal analysis," in Fractal Geometry and Applications: A Jubilee of Benoit Mandelbrot, vol. 72.2 of Proceedings of Symposia in Pure Mathematics, pp. 91151, American Mathematical Society, Providence, Rhode Island, 2004.

[13] M. Clausel, Quelques notions d'irrégularité uniforme et ponctuelle: le point de vue ondelettes, University of Paris XII, le point de vue ondelettes. $\mathrm{PhD}$ thesis, 2008.

[14] A. Khintchine, "Über einen Satz der Wahrscheinlichkeitsrechnung," Fundamenta Mathematicae, vol. 6, pp. 9-20, 1924.

[15] P. Mörters and Y. Peres, Brownian motion, vol. 30, Cambridge University Press, 2010.

[16] L. Caffarelli, R. Kohn, and L. Nirenberg, "Partial regularity of suitable weak solutions of the Navier-Stokes equations," Communications on Pure and Applied Mathematics, vol. 35, no. 6, pp. 771-831, 1982.

[17] M. Fekete, "On the distribution of roots of algebraic equations with integral coefficients (Über die Verteilung der Wurzeln bei gewissen algebraischen Gleichungen mit ganzzahligen Koeffizienten)," Mathematische Zeitschrift, vol. 17, no. 1, pp. 228-249, 1923.

[18] Z. Ditzian, "Multivariate Bernstein and Markov inequalities," Journal of Approximation Theory, vol. 70, no. 3, pp. 273-283, 1992.
[19] R. A. DeVore and R. C. Sharpley, "Maximal functions measuring smoothness," Memoirs of the American Mathematical Society, vol. 47 , no. 293, 1984.

[20] J. A. Brudnyı̆, "A multidimensional analog of a theorem of whitney," Mathematics of the USSR - Sbornik, vol. 11, no. 2, pp. 157-170, 1970.

[21] I. Daubechies, Ten Lectures on Wavelets, SIAM, Philadelphia, Pa, USA, 1992.

[22] Y. Meyer, Ondelettes et opérateurs: Ondelettes, vol. 1, Hermann, 1990.

[23] S. Mallat, A Wavelet Tour of Signal Processing, Academic Press, San Diego, Calif, USA, 1999.

[24] I. Daubechies, "Orthonormal bases of compactly supported wavelets," Communications on Pure and Applied Mathematics, vol. 41, no. 7, pp. 909-996, 1988.

[25] S. Jaffard, "Pointwise smoothness, two-microlocalization and wavelet coefficients," Publicacions Matemàtiques, vol. 35, no. 1, pp. $155-168,1991$.

[26] S. Jaffard and Y. Meyer, "On the Pointwise Regularity of Functions in Critical Besov Spaces," Journal of Functional Analysis, vol. 175, no. 2, pp. 415-434, 2000.

[27] D. W. Marquardt, "An algorithm for least-squares estimation of nonlinear parameters," Journal of the Society For Industrial \& Applied Mathematics, vol. 11, no. 2, pp. 431-441, 1963.

[28] T. Kleyntssens and S. Nicolay, "A refinement of the Sv-based multifractal formalism," In press. 


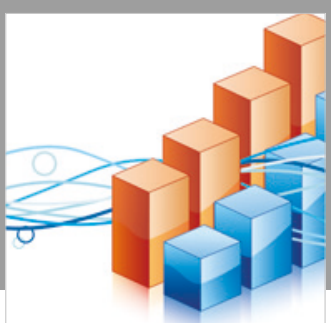

Advances in

Operations Research

\section{-n-m}
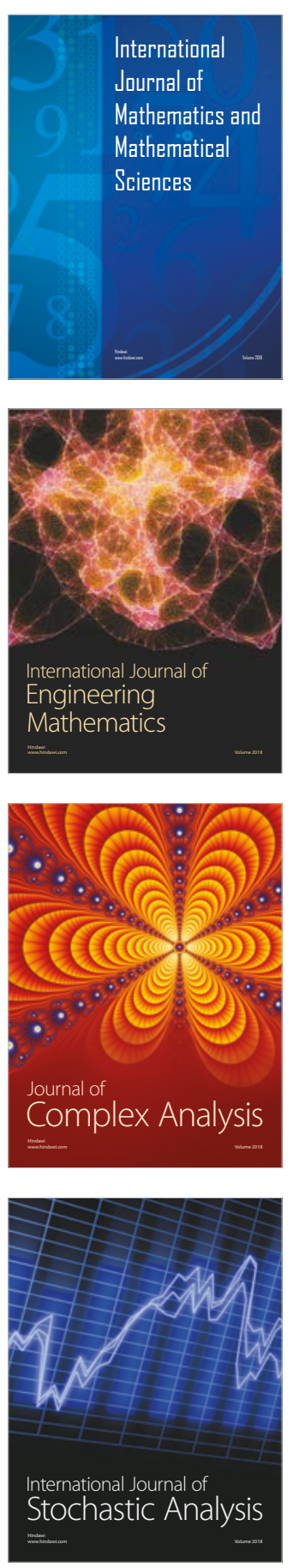
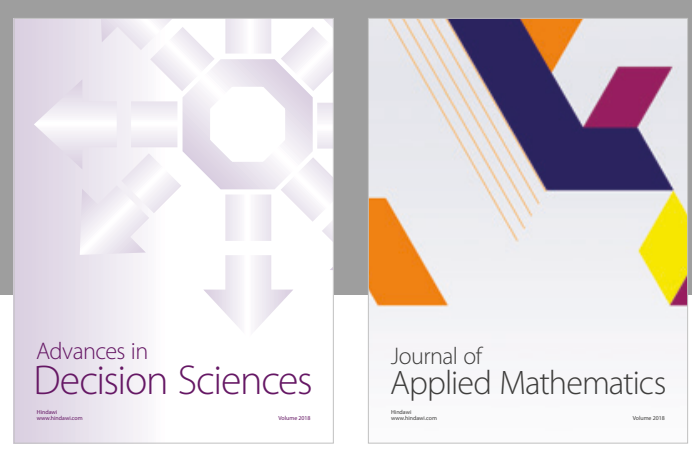

Journal of

Applied Mathematics
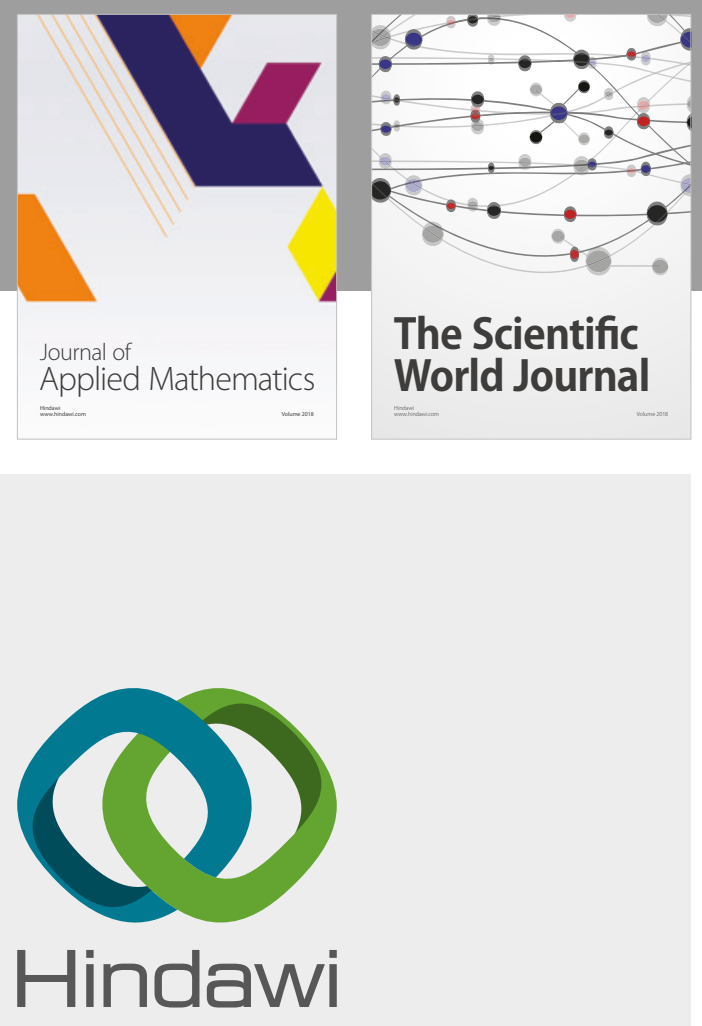

Submit your manuscripts at

www.hindawi.com

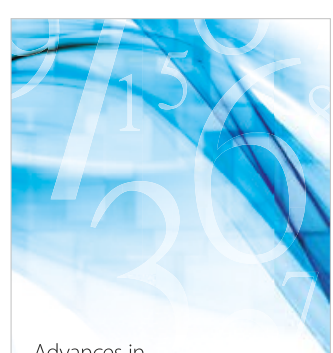

Advances in
Numerical Analysis
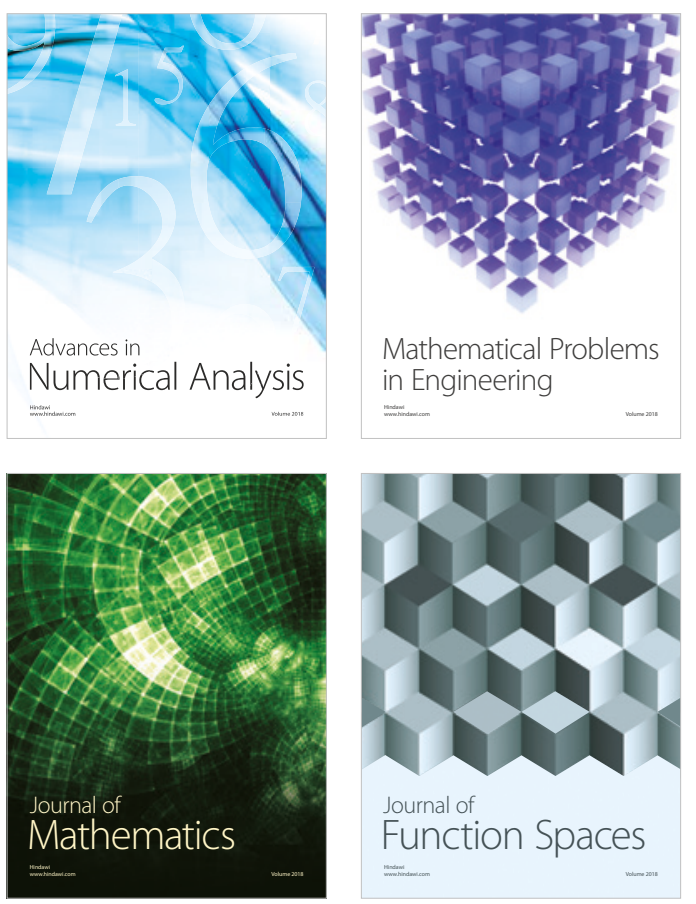

Mathematical Problems in Engineering

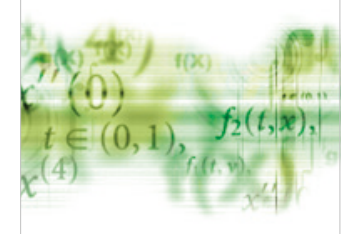

International Journal of

Differential Equations

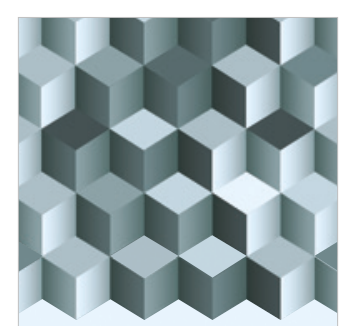

Journal of

Function Spaces

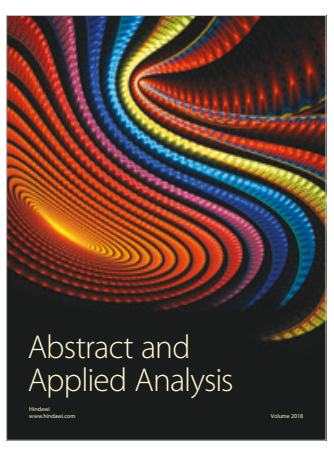

The Scientific

World Journal

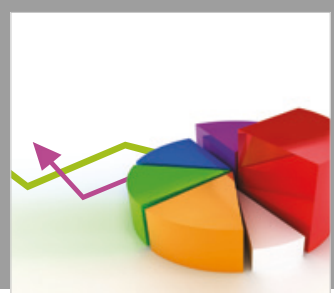

Journal of

Probability and Statistics
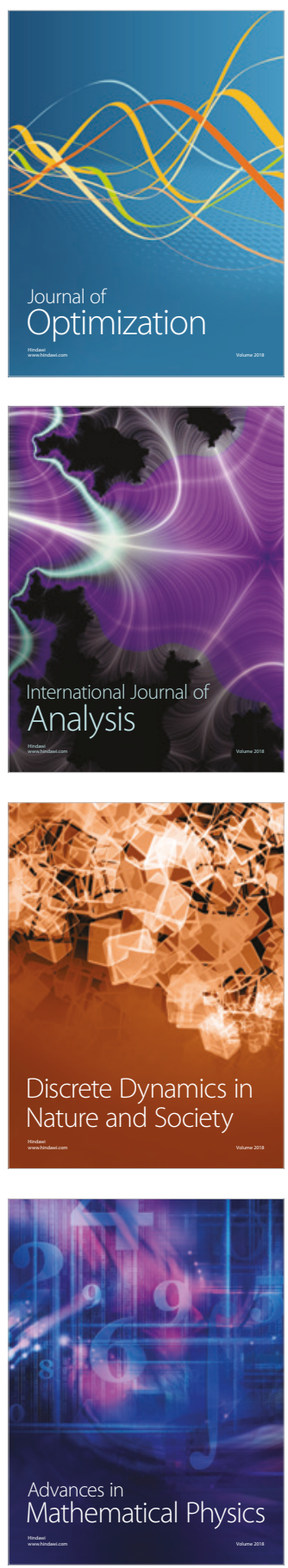